\title{
Integrated Properties of Mass Segregated Star Clusters
}

\author{
E. Gaburov ${ }^{1,2}$ and M. Gieles ${ }^{3}$ \\ ${ }^{1}$ Sterrenkundig Instituut "Anton Pannekoek", University of Amsterdam \\ egaburov@science.uva.nl \\ ${ }^{2}$ Section Computational Science, University of Amsterdam \\ ${ }^{3}$ European Southern Observatory, Casilla 19001, Santiago 19, Chile \\ email: mgieles@eso.org
}

\begin{abstract}
In this contribution we study integrated properties of dynamically segregated star clusters. The observed core radii of segregated clusters can be $50 \%$ smaller than the "true" core radius. In addition, the measured radius in the red filters is smaller than those measured in blue filters. However, these difference are small $(\lesssim 10 \%)$, making it observationally challenging to detect mass segregation in extra-galactic clusters based on such a comparison. Our results follow naturally from the fact that in nearly all filters most of the light comes from the most massive stars. Therefore, the observed surface brightness profile is dominated by stars of similar mass, which are centrally concentrated and have a similar spatial distribution.
\end{abstract}

Keywords. galaxies: star clusters

Mass segregation in star clusters is often observed from radial variations in stellar mass function (de Grijs et al. 2002a; de Grijs et al. 2002b; Kim et al. 2006). These methods are subject to biases and selection effects, such as incompleteness and blending and can, therefore, not be applied to clusters more distant than the Magellanic Clouds. Such extragalactic star clusters can only be studied through their integrated properties (Bastian et al. 2007; McCrady, Graham \& Vacca 2005). In this contribution we present simulated observations of integrated properties of dynamically segregated young star clusters.

We present a semi-analytical model of star clusters based on a simple analytical description of the mass function at different radii from the cluster centre $(r)$. Based on both observations (Kim et al. 2006) and $N$-body simulations (Portegies Zwart et al. 2007; Gaburov, Gualandris \& Portegies Zwart 2007) of young star clusters, we model the mass function in the following way. For $r<r_{\mathrm{hm}}$, with $r_{\mathrm{hm}}$ the half-mass radius, the mass function is

$$
g\left(m, r<r_{\mathrm{hm}}\right) \propto\left\{\begin{array}{ll}
m^{\alpha_{0}}, & \text { if } m<\mu=2\langle m\rangle \\
\mu^{\alpha_{0}}\left(\frac{m}{\mu}\right)^{\alpha(r)}, & \text { otherwise. }
\end{array},\right.
$$

whereas for $r>r_{\mathrm{hm}}$ it has power-law form with a constant slope $\alpha_{\mathrm{hm}}, g\left(m, r>r_{\mathrm{hm}}\right) \propto$ $m^{\alpha_{\mathrm{hm}}}$. Here, $\langle m\rangle$ and $\alpha_{0}$ are the mean mass and the slope of the initial mass function respectively, and $\alpha(r)$ is the index at the high-mass end which is a function of distance to the cluster centre. We choose $\alpha(r)=\alpha_{\text {inf }}+\left(\alpha_{c}-\alpha_{\text {inf }}\right)\left(1+(r / \epsilon)^{\delta}\right)$. The parameter $\alpha_{c}$ determines the degree of mass segregation, while parameters $\epsilon$ and $\delta$ define shape of $\alpha(r)$. The two other parameters, $\alpha_{\mathrm{hm}}$ and $\alpha_{\mathrm{inf}}$, are determined by constraints that the cluster integrated mass function results in IMF, and that the mean mass is continuous at $r_{\mathrm{hm}}$.

Given the mass function, we can calculate the mean stellar mass, $\mu(r)$, as well as the luminosity profile in different filters, $L_{\lambda}(r)$, as a function of $r$. To this end we use Padova isochrones (Girardi et al. 2002). For a given density profile, $\rho(r)$, we can obtain 

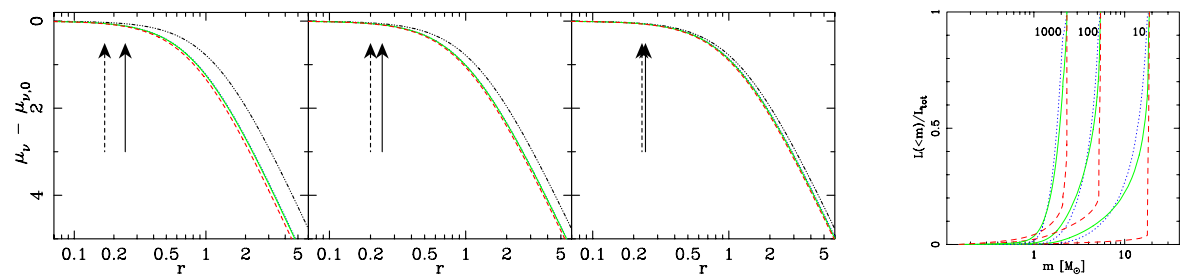

Figure 1. Normalised surface brightness profiles (left panel) of a star cluster as a function of its age. The dash-dot-dot-dot line is the surface brightness profile of a non-segregated cluster, whereas the solid, dotted and dashed lines display the profiles for mass segregated clusters in the $V, U$ and $K$ filters, respectively. The dashed and solid arrows show the core radius of segregated $(U$-filter) and non-segregated clusters respectively. The cumulative luminosity functions (right panel) are computed from a single stellar population with a power-law mass function. The numbers left of the lines show the age of the population in Myr.

spatial luminosity profile in a desired filter by using the following conversion $L_{\lambda}(r)=$ $\rho(r) \cdot\left[\lambda_{\lambda}(r) / \mu(r)\right]$. As a test case, we choose $\rho(r)$ to be an EFF profile (Elson, Fall \& Freeman 1987). The resulted surface brightness profiles are fitted to a projected EFF profile in order to obtain the core radius and power-law index.

In the case of non-segregated clusters, light distribution traces the density distribution, which is not the case for segregated star clusters. In the left panel of Fig. 1, we show the surface brightness profiles in different filters compared to surface brightness profile of non-segregated cluster. We notice that the observed core radius in segregated clusters is smaller by roughly $50 \%$ compared to the core radius of non-segregated cluster with the same density profile. Moreover, the former one is never larger than the latter one for ages $\lesssim 1$ Gyr. In addition, the difference between core radii in $U$ - and $K$-filters is smaller than $10 \%$, and it is larger for younger clusters.

Assuming a power-law mass function, we show the cumulative luminosity function in different filters in the right panel of Fig. 1. In older clusters most of the light comes from stars with similar masses which have light-to-mass ratio close to unity. Therefore, the light distribution approximates well the density distribution, and the core radii in different filters are nearly the same. In the case of young star clusters, however, the most massive stars dominate the $K$-filter, whereas stars with half the turn-off mass dominate the $U$-filter. Combined with the fact that light-to-mass ratio of these stars is notable large than unity, we expected that core radius is smaller than that of unsegregated star cluster. In addition, the core radius in $K$-filter is smaller than the core radius in $U$-filter. However, we do not expect this difference to be large, as the stars which differ in mass by a factor of two do not have significantly different spatial distribution.

Acknowledgements: We thank Nate Bastian and Søren Larsen for helpful discussions. This work is supported by NWO under grant \#635.000.303.

\section{References}

Bastian, N., Konstantopoulos, I., Smith, L. J., Trancho, G., Westmoquette, M. S., \& Gallagher, J. S. 2007, MNRAS, 379, 1333

Elson, R. A. W., Fall, S. M., \& Freeman, K. C. 1987, ApJ, 323, 54

Gaburov, E, Gualandris, A., \& Portegies Zwart, S. 2007, arXiv/0707.0406

Girardi, L., Bertelli, G., Bressan, A., Chiosi, C., Groenewegen, M. A. T., Marigo, P., Salasnich, B., \& Weiss, A. 2002 AESA, 391, 195

de Grijs, R., Johnson, R. A., Gilmore, G. F., \& Frayn, C. M., 2002a MNRAS, 331, 228

de Grijs, R., Gilmore, G. F., Johnson, R. A., \& Mackey, A. D., 2002b MNRAS, 331, 245

Kim, S. S., Figer, D. F., Kudritzki, R. P., \& Najarro, F. 2006, ApJ, 653, 113

McCrady, Na., Graham, J. R., \& Vacca, W. D., 2005 ApJ, 621, 278

Portegies Zwart, S., Gaburov, E., Chen, H.-C. \& Gürkan, M. A. 2007, MNRAS, 378, 29 